Tohoku J. exp. Med., 1976, 120, 43-51

\title{
Malignant Lymphoma Initiated with Malabsorption Syndrome Due to Isospora belli Infection and Lymphocytosis
}

\author{
Masamichi Ohtaki, Yutchi Michimata, Takashiro Suzuki, \\ Kosuke Oikawa, Masatsugu Mikami, Seiju Onodera and \\ KaORU Yoshinaga \\ The Second Department of Internal Medicine, Tohoku \\ University School of Medicine, Sendai
}

Ohtakt, M., Michimata, Y., Suzuki, T., Olkawa, K., Mikami, M., Onodera, S. and Yoshinaga, K. Malignant Lymphoma Initiated with Malabsorption Syndrome Due to Isospora belli Infection and Lymphocytosis. Tohoku J. exp. Med., 1976, 120 (1), 43-51 — A 47-year-old man had diarrhea in 1965. Four years later, malabsorption syndrome was diagnosed and the patient was found to have mild lymphocytosis. Abdominal lymphoma was suspected, but exploratory laparotomy was normal except for partial villous atrophy of small intestine and slightly enlarged mesenteric lymphnodes which were normal microscopically. In vitro lymphocyte blastformation with phytohemagglutinin was depressed markedly throughout the course and the result predicted the developement of malignancy of the lymphocytic system. Infection of Isospora belli was found thereafter, and sul. famethoxazole was quite effective for diarrhea. In August, 1974, he noticed cervical lymphadenopathy for the first time and it was diagnosed as undifferentiated type of malignant lymphoma. He died in December, 1974. In this case diarrhea was most probably caused by the intestinal infection of Isospora belli without obvious lymphoma. The symptom was swept away by peroral sulfamethoxazole. In this patient coccidiosis was presumably induced and prolonged by suppression of cellular immunity which might have already begun to progress at the onset of diarrhea. ___ - malignant lymphoma; lymphocytosis; plant agglutinin; coccidiosis and malabsorption syndrome

It is well known that malabsorption syndrome due to diarrhea may be caused by abdominal malignant lymphoma, Hodgkin's disease and $\alpha$-chain disease. But the mechanism of diarrhea in these diseases is not fully clarified as yet and there is controversy as to whether malignant lymphoma itself causes diarrhea or not (Rosenberg et al. 1961; Gough et al. 1962; Ansari and Silvis 1971; Novis et al. 1971).

On the other hand, prolonged infections of Isospora belli, coccidiosis, were reported sporadically (Brandborg et al. 1970; Syrkis et al. 1975), but the descriptions about the immunological state of the patients were inadequate; namely, whether there was depression of humoral or cellular immunity or complication of malignant lymphomas is not clear.

Received for publication, April 9, 1976.

Request for reprint should be addressed to Dr. M. Ohtaki whose present adress is Department of Internal Medicine, Sendai Municipal Hosptial, Ichibancho, Sendai 980, Japan 
Recently, we experienced a case of malabsorption syndrome with terminal malignant lymphoma. The symptom of the present case started with diarrhea, and mild lymphocytosis was found 4 years after the onset of diarrhea. Coccidiosis was found to be the cause of diarrhea, and sulfamethoxazole was effective. Lymphadenopathy appeared terminally, and an undifferentiated type of malignant lymphoma was diagnosed. Our case is considered to demonstrate correlations among malabsorption syndrome, coccidiosis, depressed cellular immunity and malignant lymphoma.

\section{Case History and Results of Examinations}

Past history. I.S., a 47-year-old man suffered from malaria and Dengue fever at age 22 in the Philippines and received quinine therapy for 5 years. Otherwise he had been healthy.

Present illness. He developed mild diarrhea in 1965 but did not need medical care for several years, until he had severe diarrhea and became emacinated, the body weight falling from $65 \mathrm{~kg}$ to $47 \mathrm{~kg}$. He was, therefore, admitted in October, 1970. On physical examination he was emacinated moderately but there were no other special findings. Neither lymphadenopathy nor hepatosplenomegaly was found. Urine and stool: Urine protein and glucose were not detectable. Stool was bulky, pale, frothy, foul-smelling and greasy. The occult blood test was negative. No parasitic ova were noted. Fat in stool was $14.6 \mathrm{~g} /$ day (normal range below $5 \mathrm{~g} /$ day), ${ }^{131} \mathrm{I}$ PVP fecal excretion $1.4 \%$ (normal range below $1 \%$ ), and $\mathrm{D}$-xylose test $0.4 \mathrm{~g}$ (normal range over $1.5 \mathrm{~g}$ ). Peripheral blood counts: RBC $340 \times 10^{4}, \mathrm{Hb} 10.7 \mathrm{~g} / 100 \mathrm{ml}$ and WBC 17,600 (neutrophils $15 \%$, basophils $0 \%$, eosinophils $1 \%$, monocytes $1 \%$, lymphocytes $83 \%$ ). Absolute lymphocytosis in peripheral blood was found and repeated counting revealed that the absolute number of lymphocytes was $9,000-17,000 / \mathrm{mm}^{3}$ including $23-32 \%$ of atypical, notched, twinning nuclear lymphocyte (Fig. 1). PAS reaction of lymphocyte was

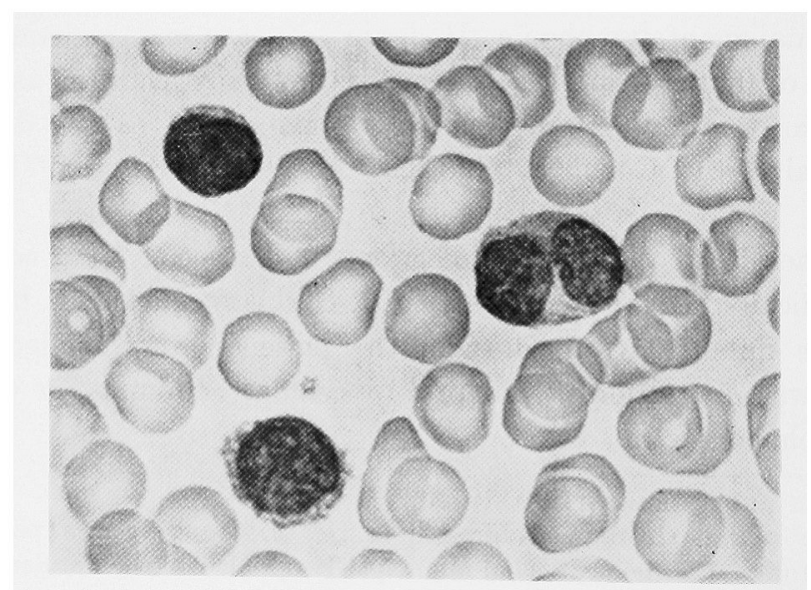

Fig. 1. Peripheral blood film showing twinning nuclear lymphocyte. 
almost always negative. Bone marrow smear showed slight eosinophilia (12\%) and mild lymphocytosis $(32.6 \%)$, but no abnormality in granulocytes or erythrocytes series. Liver function: SGOT 39, SGPT 43, alkaline phosphatase $8.3 \mathrm{KA}$ unit, and BSP $3.0 \%(30 \mathrm{~min})$. Blood glucose levels on the glucose tolerance test (100 g glucose perorally) were: before $70 \mathrm{mg} / 100 \mathrm{ml}, 30 \mathrm{~min} 74 \mathrm{mg} / 100 \mathrm{ml}, 60$ $\min 100 \mathrm{mg} / 100 \mathrm{ml}, 90 \mathrm{~min} 80 \mathrm{mg} / 100 \mathrm{ml}$, and $120 \mathrm{~min} 72 \mathrm{mg} / 100 \mathrm{ml}$, indicating a flat curve. Serum amylase was 3-64 units and urine amylase 32-64 units. Gastric juice showed achlorhydria. Serum total protein measured $7.4 \mathrm{~g} / 100 \mathrm{ml}$ (albumin $59.1 \%, \alpha_{1}$-globulin $3.1 \%, \alpha_{2}$-globulin $9.4 \%, \beta$-globulin $8.2 \%, \gamma$-globulin $20.2 \%$ ). Immunoglobulin concentration was normal (Fig. 2) and there was no abnormal bow on immunoelectrophoresis. Serum total cholesterol was $110 \mathrm{mg} /$ $100 \mathrm{ml}$, triglycelide $74 \mathrm{mg} / 100 \mathrm{ml}$, phospholipid $147 \mathrm{mg} / 100 \mathrm{ml}$, serum $\mathrm{Na} 140$ $\mathrm{mEq} /$ liter, K $4.9 \mathrm{mEq} / \mathrm{liter}, \mathrm{Cl} 104 \mathrm{mEq} /$ liter and BUN $11 \mathrm{mg} / 100 \mathrm{ml}$. The RA test, CRP and STS were negative. ASO was 125 Todd units. Mantoux reaction was $10 \times 10 \mathrm{~mm}$ in October, 1969 , and $6 \times 6 \mathrm{~mm}$ in October, 1974. ECG was normal. On chest $x$-ray no abnormality was found in the lung fields or hilus.

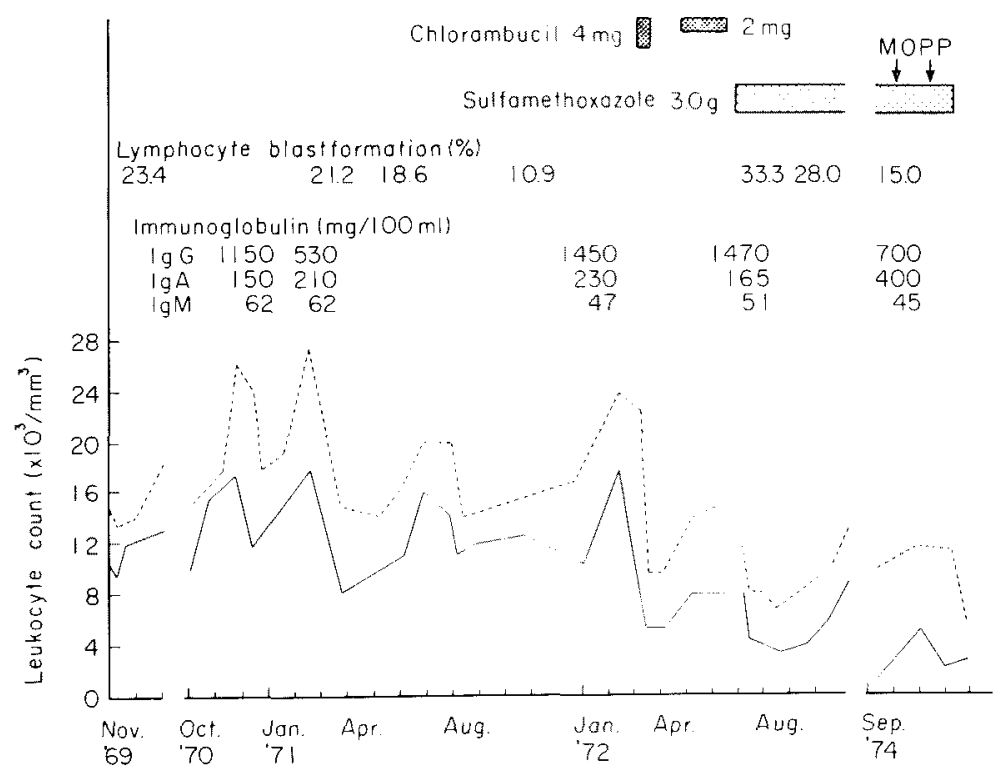

Fig. 2. Course after admission to Tohoku University Hospital. In vitro lymphocyte blastformation with phytohemagglutinin was decreased markedly compared with normal range $71.0-88.0 \%$. -...., WBC; - - absolute peripheral blood lymphocyte count.

Malabsorption syndrome was evident from these data, but its cause was uncertain. The cause of lymphocytosis was also obscure. Lymphangiographically, there was no abnormality (Fig. 3a). Gluten free diets were tried for diarrhea, but no improvement was gained and celiac disease was ruled out. Tetracycline, ampicillin, and chloramphenicol were then tried one after another but there was 


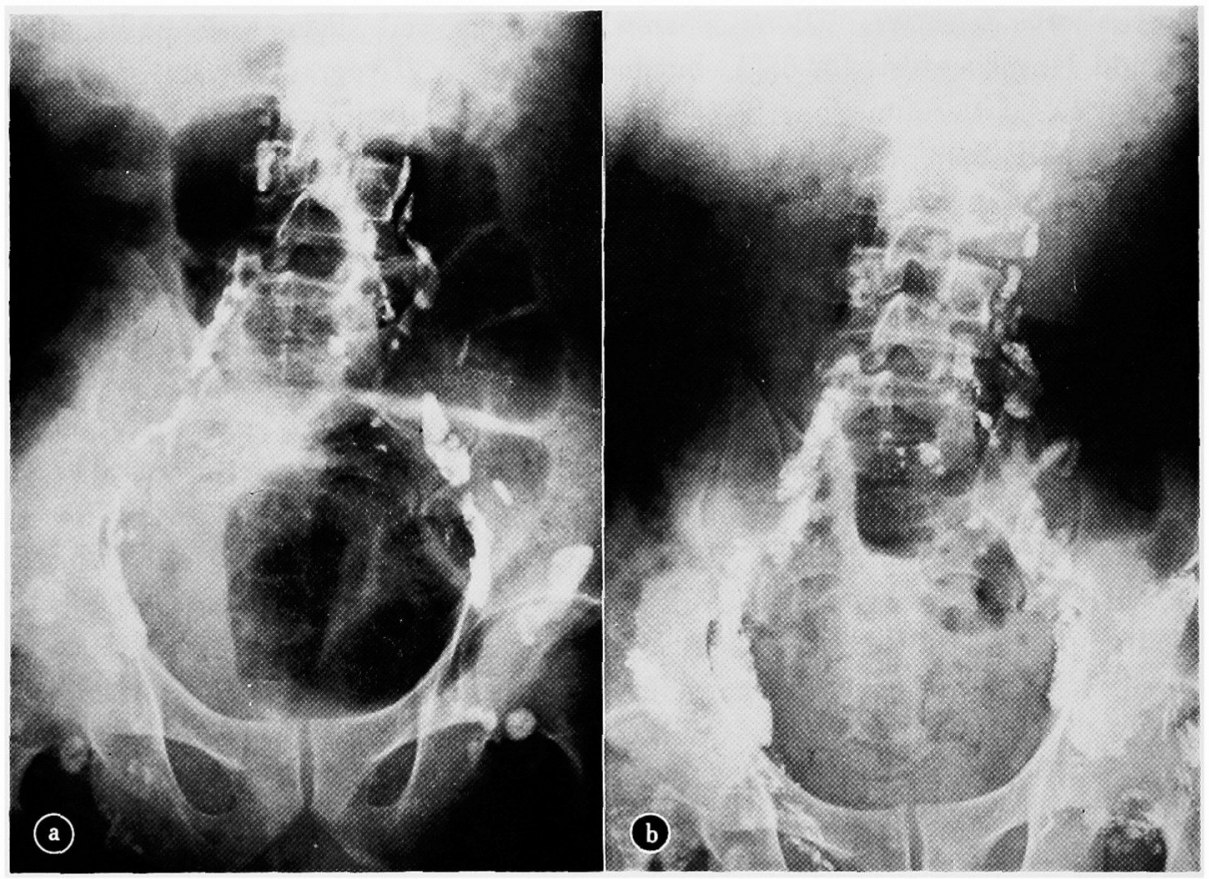

Fig. 3a. Normal pattern of lymphangiogram, October, 1970.

Fig. 3b. Abnormally enlarged pattern of lymphangiogram, August, 1974.

no effect for diarrhea. In vitro lymphocyte blastformation with phytohemagglutinin was decreased markedly to $10.9-33.3 \%$ (normal range $71.0-88.0 \%$ ) (Fig. 2), suggesting the existence of malignancy in the lymphocytic system. Blastformation was influenced neither with wash of lymphocyte nor by exchanging patient plasma with normal plasma.

Peroral duodenal biopsy demonstrated partial villous atrophy of the mucous membrane (Fig. 4) with some infiltration of plasma cells and eosinophils. Exploratory laparotomy was performed in April, 1971 for suspected WDHA syndrome because of possible abnormality in abdominal angiography and also for possible abdominal lymphoma. But we could find neither of them. Mesenteric lymphnodes were slightly enlarged, but they were normal on microscopic examination (Fig. 6). Findings of biopsied specimens from several portions of the small bowel were the same as those of duodenal biopsy. There were no findings suggesting malignant lymphoma or $\alpha$-chain disease.

The patient was treated with $4 \mathrm{mg}$ /day of chlorambucil with the purpose of reducing peripheral lymphocytes in February, 1972 (Fig. 2). Lymphocyte count decreased to the normal level, but diarrhea continued. Through reexamination of the biopsied specimen of the small bowel with PAS stain (Fig. 5) and repeated examination of duodenal juice for parasitic ova, Isospora belli infection was diagnosed. Peroral administration of sulfamethoxazole $3 \mathrm{~g} /$ day was absolutely effective for diarrhea, and oocytes in feces disappeared in 10 days. 


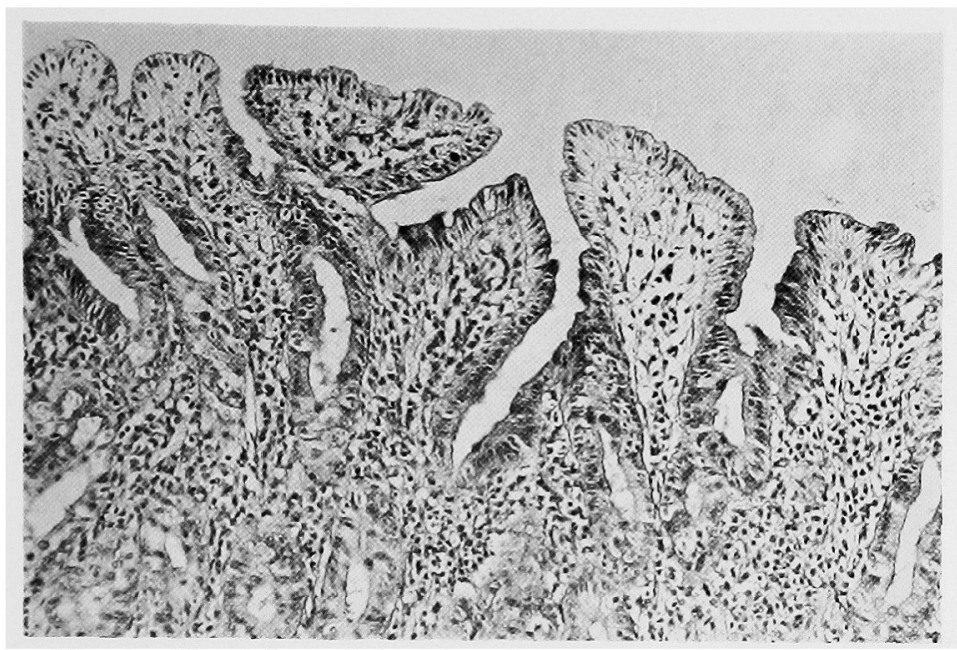

Fig. 4. Biopsied small intestine showing partial villous atrophy.

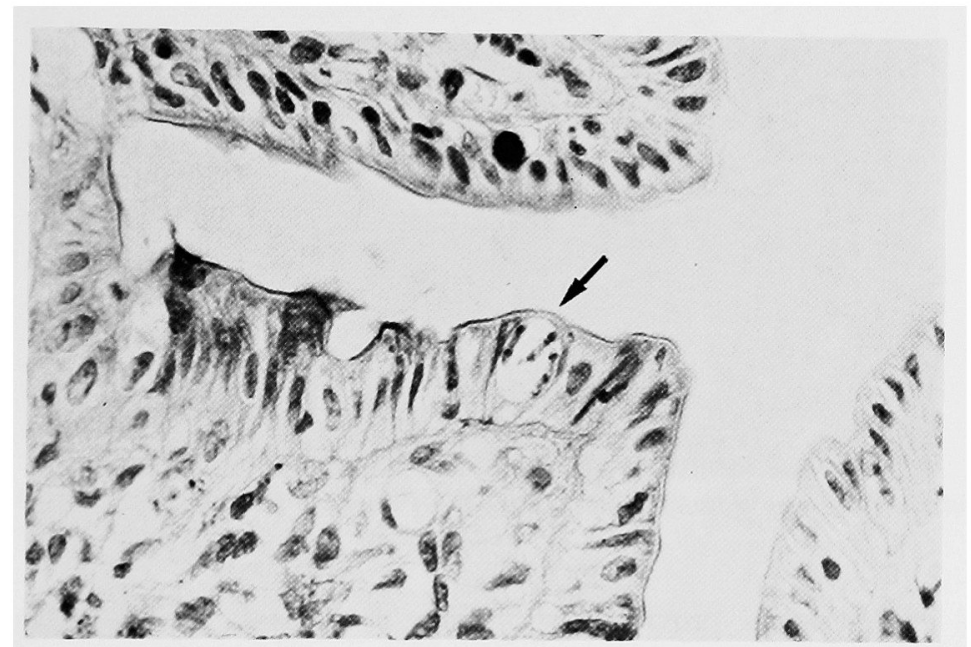

Fig. 5. Biopsied small intestine stained with PAS. The arrow indicates merozoite of Isospora belli.

Lymphocyte count of the peripheral blood decreased somewhat as shown in Fig. 2, but lymphocyte response to phytohemagglutinin remained at a low level as before.

General conditions of the patient improved markedly and he was discharged in October, 1972 and continued to take sulfamethoxazole. In August, 1974, he noticed swelling of the cervical lymphnode and biopsy of the node was performed. Histologically, an undifferentiated type of malignant lymphoma was diagnosed (Fig. 7). The picture of the lymphnode was quite different from the former histology. The lymphnode had lost its normal architecture and the indivisual 


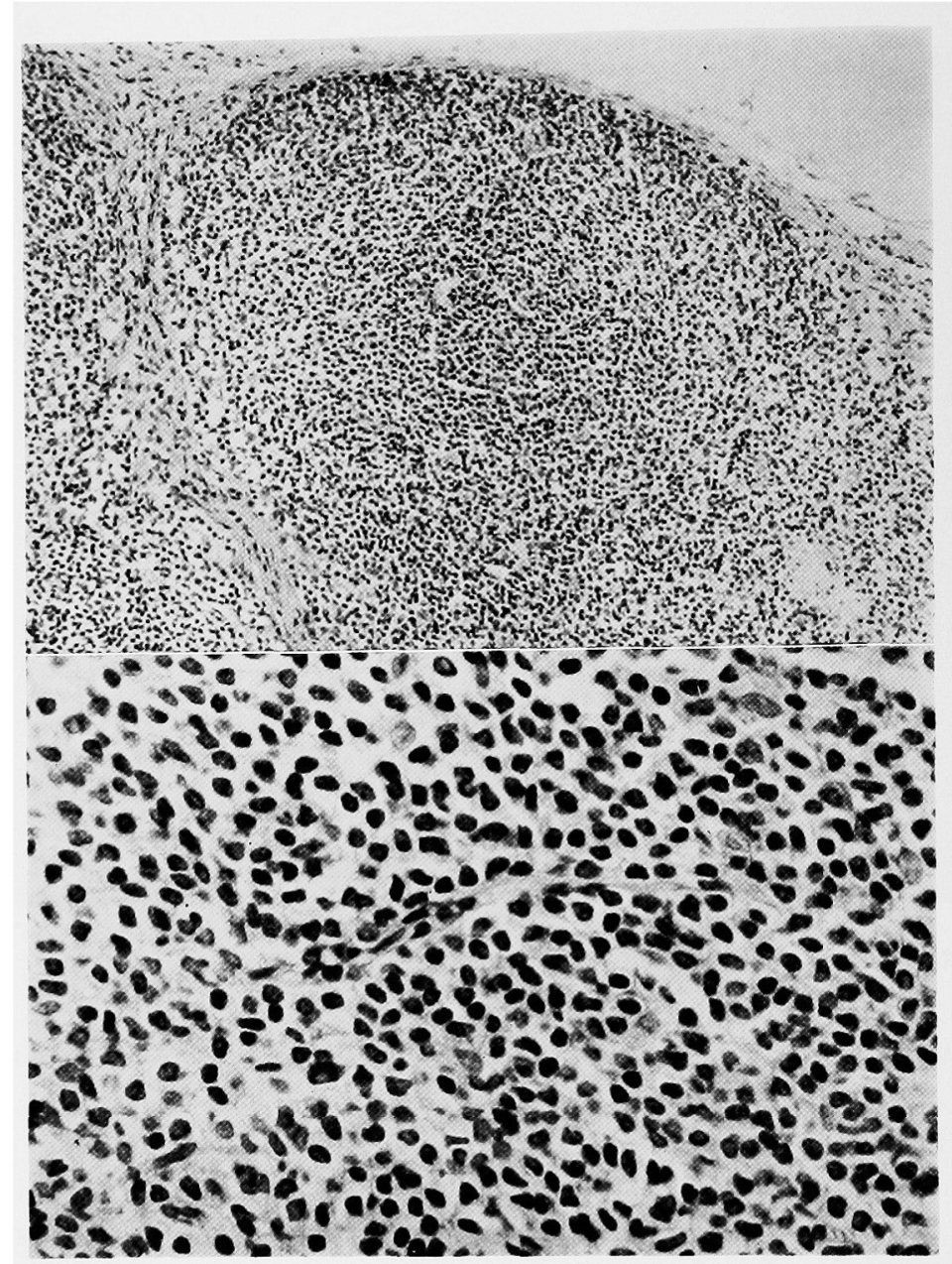

Fig. 6. Mesenteric lymphnode, April, 1971, showing almost normal structure.

cells were enlarged and provided with wide cytoplasma and a polygonal clear nucleus with nucleolus. Mitosis were seen frequently.

Lymphangiogram indicated enlarged abdominal lymphnodes and the pattern of malignant lymphoma (Fig. 3b). Peripheral blood lymphocyte counts were not increased as before and lymphocytes with an abnormally notched, twinning nuclei were sparse.

In vitro lymphocyte blastformation with phytohemagglutinin still remained at a low level. Mantoux reaction became negative. Phytohemagglutinin-P intracutaneous reaction was $6 \times 6 \mathrm{~mm}$ (normal, above $10 \times 10 \mathrm{~mm}$ ). MOPP therapy was introduced immediately, but its effect on lymphoma was only temporary, and the patient expired in December, 1974. 


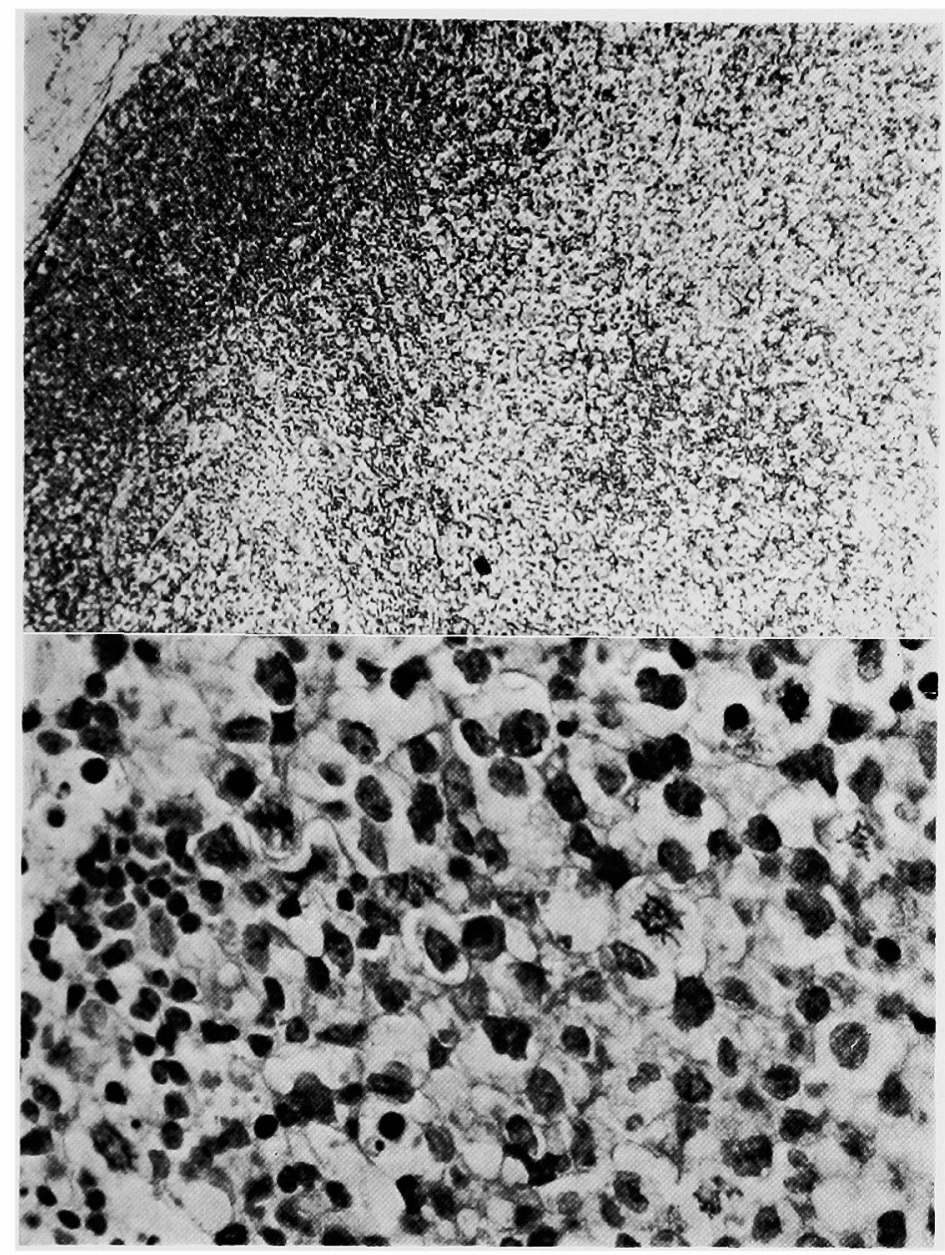

Fig. 7. Cervical lymphnode, August, 1974, showing histology of an undifferentiated type of malignant lymphoma.

\section{Discussion}

Many underlying diseases are mentioned as the causes of malabsorption syndrome due to infiltrative processes of the small intestine, such as regional enteritis, amyloidosis, scleroderma, malignant lymphoma, tropical sprue and infectious enteritis. Although malabsorption syndrome complicating malignant lymphoma was also reported frequently, the relationship between malignant lymphoma and diarrhea is still controversial. Namely, some reported that malignant lymphoma itself causes malabsorption (Novis et al. 1971), but others stated that cases of intestinal involvement of malignant lymphoma were seldom associated with diarrhea and that it was difficult to consider that malignant lymphoma caused diarrhea (Rosenberg et al. 1961).

Certainly, it is questionable that malignant lymphoma in some portion of the 
intestine causes extensive intestinal villous atrophy. On the other hand, various intervals of celiac disease are reported to precede malignant lymphoma, and celiac disease is thought as a prelymphomatous state (Ansari and Silvis 1971).

The infection of Isospora belli, a coccidiosis, is considered generally as a tropical and self-limiting disease, but occasionally it becomes prolonged and severe (Brandborg et al. 1970, Syrkis et al. 1975). The cause of prolongation of coccidiosis is not clarified enough, but it seems rather important whether there is some underlying disease or not. Complication of Hodgkin's disease in the former report is quite interesting from the viewpoint of immunological states including humoral and cellular immunity.

Diarrhea of our case was caused by Isospora belli. Administration of sulfamethoxazole cleared the parasite, stopped diarrhea and restored the mucosa of the small intestine to a normal condition. Generally abdominal lymphoma has been mentioned as a cause of diarrhea, but the cause of diarrhea of our case could be traced back one step further.

Humoral immunodeficiency accompanied by malabsorption syndrome was also reported in IgA deficiency (Gelzayd et al. 1971) or $\alpha$-chain disease (Henry et al. 1974). However, the quality and quantity of immunoglobulins of our case were normal. Lymphocyte response to phytohemagglutinin as an expression of cellular immunity had been depressed throughout the course of the present case even before the appearance of malignant lymphoma. In some investigations it was reported that the lymphocyte response to phytohemagglutinin was depressed in celiac disease (Hobbs et al. 1969) and Whipple's disease (Maxwell et al. 1968).

The course of our case is quite unusual for malignant lymphoma. The patient developed diarrhea as an initial symptom and had lymphocytosis 5 years later. Morphologically, some lymphocytes had notched, twinning deformity of the nucleus and the lymphocytes in this patient did not respond to phytohemagglutinin. At that time, there was no lymphadenopathy and the architecture of the mesenteric lymphnode was quite normal. It was impossible to arrive at the definite diagnosis of malignant lymphoma at that time. Four years later, that is 9 years after the onset of diarrhea and 4 months before death, cervical lymphadenopathy and splenomegaly were noticed for the first time. The diagnosis of undifferentiated type of malignant lymphoma was established definitely by lymphnode biopsy. Generally, lymphomas become leukemic in their terminal stages but the course of our case was quite the reverse concerning peripheral blood findings.

Clinical features of our case were quite atypical for malignant lymphoma and the patient had been infected with Isospora belli even before malignant lymphoma was diagnosed. It must be admitted, however, that the relation between Isospora belli and malignant lymphoma is utterly obscure. In a report of complication of coccidiosis in $\alpha$-chain disease, Henry et al. (1974) said that Isospora belli was more than a superimposed infection in an immunologically defective patient and might play an etiological role in the pathognesis of heavy chain disease. We supposed that abnormality of lymphocytes which is common in malignant lymphoma developed 
gradually and caused depression of cellular immunity. The advent of abnormal lymphocytes will really indicate the beginning of malignant lymphoma. It is further assumed that infection of Isospora belli is initiated by the depression of cellular immunity. We thought that abnormality of lymphocyte evolved progressively, and finaly it precipitated undifferentiated malignant lymphoma.

\section{Acknowledgment}

We are indebted to Ass. Prof. Haruki Wakasa for his kind help for pathological findings, Dr. Osamu Masamune for gastrointestinal absorption tests and Dr. Masanori Mita for endoscopic examination.

\section{References}

1) Ansari, A. \& Silvis, S.E. (1971) Developement of malignant lymphoma during the course of adult celiac diease. Amer. J. Gastroent., 55, 482-488.

2) Brandborg, L.L., Goldberg, S.B. \& Breidenbach, W C. (1970) Human coccidiosis - A possible cause of malabsorption. The life cycle in small-bowel mucosal biopsies as a diagnostic feature. New Eng. J. Med., 283, 1306-1313.

3) Gelzayd, E.A., MeCleery, J.L., Melnyk, C.S. \& Kraft, S.C. (1971) Intestinal malabsorption and immunoglobulin deficiency. Arch. intern. Med., 127, 141-147.

4) Gough, K.R., Read, A.E. \& Naish, J.M. (1962) Intestinal reticulosis as a complication of idiopathic steatorrhoea. Gut, 3, 232-239.

5) Henry, K., Bird, R.G. \& Doe, W.F. (1974) Intestinal coccidiosis in a patient with alpha-chain disease. Brit. med. J., 1, 542-543.

6) Hobbs, J.R., Hepner, G.W., Douglas, A.P., Crabbé, P.A. \& Johansson, S.G.O. (1969) Immunological mystery of coeliac disease. Lancet, 2, 649-650.

7) Maxwell, J.D., Ferguson, A., McKay, A.M., Imrie, R.C. \& Watson, W.C. (1968) Lymphocytes in Whipple's disease. Lancet, 1, 887-889.

8) Novis, B.H., Banks, S., Marks, I.N., Selzer, G., Kahn, L. \& Sealy, R. (1971) Abdominal lymphoma presenting with malabsorption. Quart. J. Med., New Series, 40, 521-540.

9) Rosenberg, S.A., Diamond, H.D., Jaslowitz, B. \& Craver, L.F. (1961) Lymphosarcoma: a review of 1,269 cases. Medicine (Baltimore), 40, 31-84.

10) Syrkis, I., Fried, M., Elian, I., Pietrushka, D. \& Lengy, J. (1975) A case of severe human coccidiosis in Israel. Israel $J$. med. Sci., 11, 373-377. 